\title{
Optimization of TAC Configuration in Mobile Communication Systems: A Tabu Search Approach
}

\author{
Hyung-Woo Kang*, Hyon-Goo Kang**, Seok-Joo Koh* \\ *School of Computer Science and Engineering, Kyungpook National University, Korea \\ **Access Network Lab, SK Telecom, Korea \\ hwkang0621@gmail.com, hyongoo.kang@sk.com, sjkoh@knu.ac.kr
}

\begin{abstract}
Recently, the mobile communication has been rapidly changing the LTE-based mobile communication. In LTE-based mobile communication system, paging performance is a critical factor to be considered, a paging area is defined as TAC, which is group of cells. In this paper, we propose a new scheme for configuration of TAC to maximize the paging success rate. This scheme constructs a TAC using traffic load, TAC size and handover patterns. Then we propose the improvement algorithm using tabu search. From the performance analysis with real traffic data of Seoul in Korea, we can see that the proposed TAC configuration provides larger paging success rates than the existing TAC configuration.
\end{abstract}

Keywords - Mobile Network System, Paging, Tracking Area Code, Optimization, Tabu Search

\section{INTRODUCTION}

With the prevalence of smart phones, mobile communication has been rapidly evolved to the Long Term Evolution (LTE) $[1,2]$. In LTE-based mobile systems, the paging performance is one of the important factors to be considered $[3,4]$. The paging operation is initiated to locate a mobile user in the network, when a call request to the user arrives. In the LTE-based mobile systems, a paging area is defined by a Tracking Area Code (TAC).

In this paper, we propose a new scheme configuration of TACs to improve the paging success rate. A TAC consists of a group of cells to which a paging signal is broadcast in the gaging process. The proposed scheme is based on a mathematical optimization model for TAC configuration, in which we consider the mobility (handover) patterns of mobile users as well as the TAC size and the capacity of paging traffic. Then, we propose the improvement algorithm for TAC configuration to maximize the paging success rate, while some constraints are satisfied.

This paper is organized as follows. In Section 2, we discuss the TAC configuration and the paging operations. In Section 3, we describe an optimization model for TAC configuration and pro-posed TAC configuration algorithm. Section 4 analyzes the paging performance of the existing and proposed schemes in terms of the paging success rate, with the real traffic data. Finally, Section 5 concludes this paper.

\section{OPTIMization MODEL}

Figure 1 shows an example of TAC configuration in mobile net-work. In this figure, there are 11 TACs in a network area, and a group of cells are assigned to a TAC. TAC is encoded with a 2-bytes hexadecimal digit (e.g., 010A), in which the first byte (e.g., 01 in the example) represents the associated network area, and the second byte (04) is used to identify the $\mathrm{TAC}$ in the area.

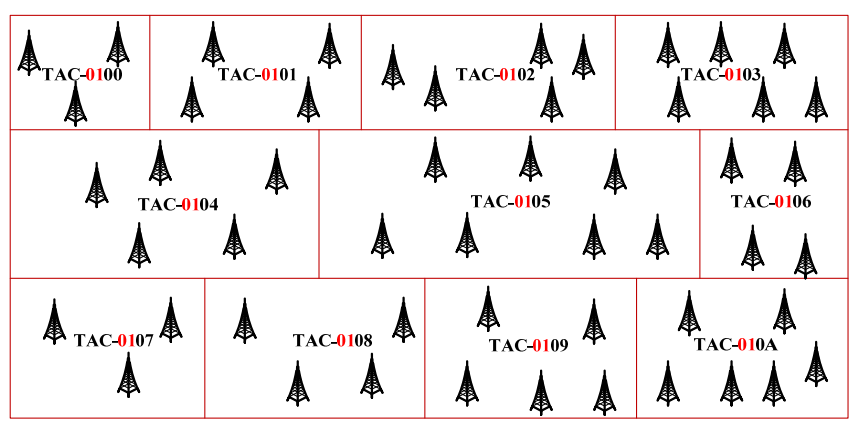

Figure 1. Example of TAC Configuration

With such TAC configuration, when a paging request to a specific user arrives, the paging operations are performed as follows. In the first paging, the paging request message (or signal) will be broadcast to the cells contained in the TAC. If the paging request fails (i.e., no response to the paging request from the mobile user), then the second paging is performed, in which the paging request will be broadcast to all of cells in the area. Therefore, it is important to optimize the TAC configuration so as to maximize the paging success rate of the first paging and thus to reduce the paging traffics generated in network.

At present, most of the mobile operators configure the TAC in an arbitrary way, in which only the geographical location information of all cells are considered and the network manager manually configures a group of cells as a TAC. That is the mobility or handover pattern was not considered in the TAC configuration. So, if the user has already moved to another cell during the dormant mode, the actual TAC of the user may be changed and thus it is likely that the first paging process fails. 
Recently, the Self-Organizing Network (SON) system is used in the LTE-based mobile communication system in order to automatically configure and manage a mobile network. SON network, which adapt itself to environment, has characteristics that self-configuration, self-service, selfknowledge, self-awareness and maintenance. In this paper, for TAC configuration, we propose the optimization algorithm which is applied on SON system. The proposed scheme is designed by considering the mobility (handover) patterns of mobile users as well as the TAC size and the capacity of paging traffic. Figure 2 shows the overview of SON system.

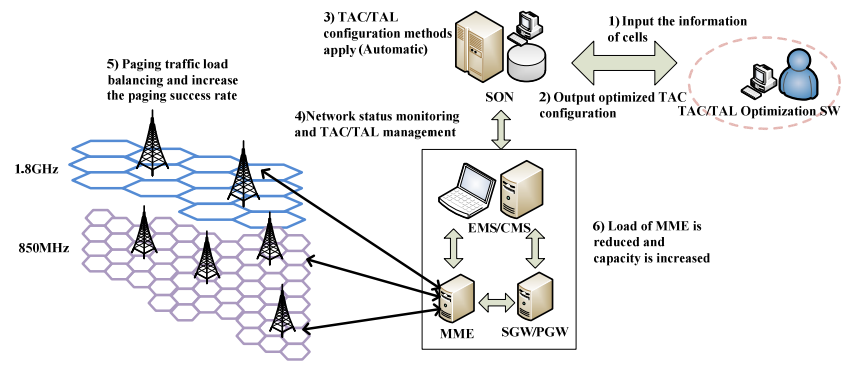

Figure 2. Overview of SON System

For TAC optimization, we first make a mathematical optimization model for TAC configuration.

$>\mathrm{h}_{\mathrm{ij}}$ : handover ratio that a user moves from cell $\mathrm{i}$ to cell $\mathrm{j}$, where $\Sigma_{\mathrm{j} \in \mathrm{N}} \mathrm{h}_{\mathrm{ij}}=1$;

$>\lambda_{\mathrm{i}}$ : average paging traffic load for cell $\mathrm{i}$, which is calculated as the number of RRC connections established in the cell;

$>\quad \mathrm{N}$ : a group of total cells in the area, with the size of $n$;

$>$ M: a group of TACs in the area, with the size of $m$;

$>\mathrm{S}_{\mathrm{TAC}}$ : the maximally allowable number of cells for a TAC;

$>\mathrm{C}_{\mathrm{TAC}}$ : the maximum paging traffic load allowable for a TAC;

$>\mathrm{x}_{\mathrm{ik}}$ : a decision variable, $\mathrm{x}_{\mathrm{ik}}=1$ if cell $\mathrm{i}$ is assigned to TAC $k, \mathrm{x}_{\mathrm{ik}}=0$ otherwise.

Based on these variables and parameters, we can make an optimization model for TAC optimization, as shown in Figure 3 .

$$
\begin{aligned}
& \text { Maximize } \quad \sum_{\mathrm{k} \in \mathrm{M}} \sum_{\mathrm{i} \in \mathrm{N}} \Sigma_{\mathrm{j} \in \mathrm{N}} \lambda_{\mathrm{i}} \times \mathrm{h}_{\mathrm{ij}} \times \mathrm{x}_{\mathrm{ik}} \times \mathrm{x}_{\mathrm{jk}} \\
& \text { Subject to (constraint conditions) } \\
& \quad \sum_{\mathrm{k} \in \mathrm{M}} \mathrm{x}_{\mathrm{ik}}=1 \text {, for all } \mathrm{i} \in \mathrm{N} \\
& \sum_{\mathrm{i} \in \mathrm{N}} \mathrm{x}_{\mathrm{ik}} \leq \mathrm{S}_{\mathrm{TAC}} \text {, for all } \mathrm{k} \in \mathrm{M} \\
& \quad \sum_{\mathrm{i} \in \mathrm{N}} \lambda_{\mathrm{i}} \times \mathrm{x}_{\mathrm{ik}} \leq \mathrm{C}_{\mathrm{TAC}} \text {, for all } \mathrm{k} \in \mathrm{M} \\
& \mathrm{x}_{\mathrm{ik}}=1 \text { or } 0 \text {, for all } \mathrm{i} \in \mathrm{N}, \mathrm{k} \in \mathrm{M}
\end{aligned}
$$

Figure 3. TAC Optimization Model

In this model, the objective function represents the PSR that is defined as the ratio of $\lambda_{\mathrm{i}} \times \mathrm{h}_{\mathrm{ij}}$ in which a user moves cell $\mathrm{i}$ to cell $\mathrm{j}$, and both $\mathrm{i}$ and $\mathrm{j}$ are assigned to the same TAC $\mathrm{k}$ (i.e., $\mathrm{x}_{\mathrm{ik}}$ $=1$ and $\mathrm{x}_{\mathrm{jk}}=1$ ).

In addition, we consider the following three constraints:

(1) TAC assignment: each cell should be assigned to one TAC;

(2) TAC size: the number of cells assigned to a TAC cannot exceed $\mathrm{S}_{\mathrm{TAC}}$;

(3) Paging traffic capacity: the paging traffic load for a TAC cannot exceed $\mathrm{C}_{\mathrm{TAC}}$.

It is noted that this optimization problem is similar to the knapsack problem, which is known as an NP-complete problem [5]. The TAC optimization problem can be reduced to the knapsack problem by considering only a single TAC (i.e., $\mathrm{m}=1$ ) and by taking only the constraints (3) and (4). Accordingly, we use heuristic algorithms such as tabu search to solve the TAC optimization problem

\section{Proposed Tabu Search}

\section{A. Overview of Tabu Search}

Tabu search is a heuristic search method and a local search strategy with a flexible memory structure [6-11]. Tabu restricts some search of neighboring solution. Local (neighbourhood) searches take a potential solution to a problem and check its immediate neghbors (that is, solutions that are similar except for one or two minor details) in the hope of finding an improved solution.

Tabu search enhances the performance of these techniques by using memory structures that describe the visited solutions or user-provided sets of rules. Tabu search uses a local or neighbourhood search procedure to iteratively move from one potential solution $x$ to an improved solution $x^{\prime}$ in the neighbourhood of $x$, until some stopping criterion has been satisfied.

The overall approach of tabu search is to avoid entrainment in cycles by forbidding or penalizing moves which take the solution, in next iteration, to points in the solution space previously visited (hence "tabu"). Tabu search always move to the best available neighborhood solution point, even if it is worse than the current solution point. The solutions are not in the tabu list, or in tabu list but satisfy aspiration condition.

In tabu search, we consider two factors that are tabu list and tabu size. Tabu list maintains a list of solution point that must be avoided (not allowed) or a list of move attributes that are not allowed. Tabu size is length of tabu list. That is, tabu size is defined that how many tabus consider. Stopping criterion is also important. According to stopping criterion, more optimized solution can be found.

\section{B. Initial TAC Configuration}

Before optimization, we think about initial TAC configuration that uses existing TAC configuration used in mobile network. Based on the really used existing TAC configuration, Tabu search algorithm performed. 


\section{Definition of Move for Tabu Search}

For improve paging success rate, proposed TAC configuration use tabu search. Move of tabu search is defined that a cell of specific TAC moves to another TAC where maximize PSR. The move is performed to all the cells in the area. This move procedure continues until a stopping criterion is satisfied.

Figure 4 shows example of move procedure.

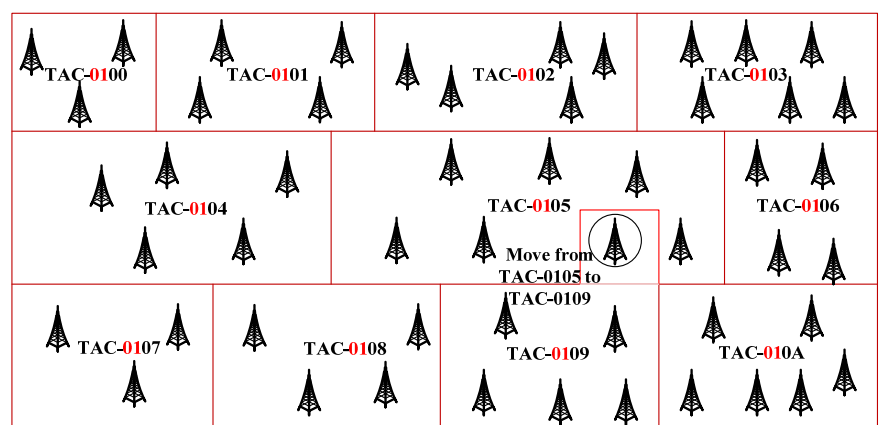

Figure 4. TAC Configuration Algorithm for Tabu Search

\section{Tabu Size and Stopping Criterion}

Tabu size is defined 50, 100, 150 and 200. That is, 50, 100, 150 and 200 cells can be stored in tabu list.

Proposed scheme is stopped if PSR doesn't improve 40 times sequentially.

\section{E. Overall Algorithms}

To solve the TAC optimization problem, we propose the improvement algorithm using tabu search. The proposed algorithm for improvement of TAC configuration is described in Figure 5.

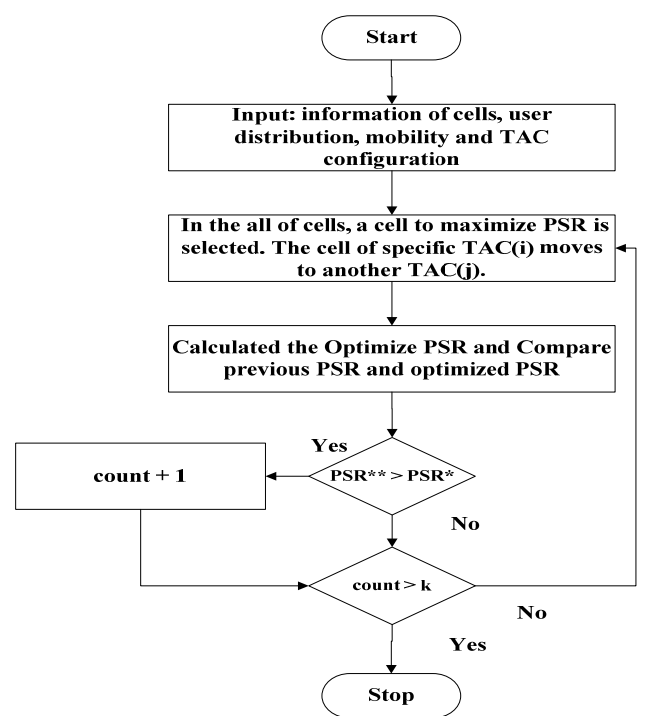

Figure 5. TAC Configuration Algorithm for Tabu Search
As input to existing TAC configuration, we consider the mobility ratio and paging traffic per cell. Based on the configuration obtained by randomly or existing configuration, we try to change the TAC of a cell to another TAC. After executing the TAC change process about all cells, we select a cell, which is maximize PSR and feasibility condition is satisfied and move it. This is one move in improvement algorithm.

After one move, the cell which is moved added in tabu list. Tabu list save the $\mathrm{m}$ cells (the $\mathrm{m}$ is celled tabu size) that is moved recently. When the next move, we don't consider the cells in the tabu list.

This improvement algorithm is repeated until if the PSR value is not improved continuously $\mathrm{k}$ times. $\mathrm{k}$ is stopping criterion and count is values of stop count. If solution PSR is lower than previous PSR, stop and count increase. The previous PSR denoted by PSR** and optimized PSR denoted by PSR* in Figure 5.

\section{IV.EXPERIMENTAL RESULTS}

\section{A. Test Network Environments}

In experiments for performance analysis, we use the realworld data of network topology, user paging traffic, and mobility rates, which are given by SK Telecom in Korea. The proposed TAC configuration scheme was applied to a target area, and we calculate the PSR values.

For experiments, the default parameter values are set as follows: $\mathrm{S}_{\mathrm{TAC}}=120, \mathrm{C}_{\mathrm{TAC}}=2100$.

\section{B. Test Results and Discussion}

In experiments, we calculate the paging success probability $(P S P)=$ PSR $/ \sum_{i \in \mathrm{N}} \lambda_{\mathrm{i}}$, which represents the ratio of successfully paged traffics over total paging traffics in the network.

Figure 6 shows change of the PSP for target area 43. Tabu size is set 200 cells. The graph shows that from the hundredth to the 11 hundredth moves for tabu search.

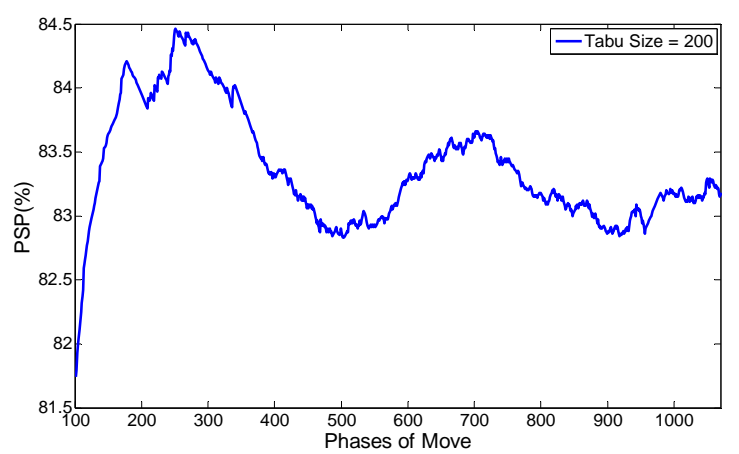

Figure 6. TAC Optimization for Tabu Search using Tabu size 200

Table I shows the results of the existing and proposed TAC configuration schemes for the target area 43, which has $n$ 
$($ total number of cells $)=1610$ and $m($ the number of TACs $)=$ 15.

TABLE 1. COMPARISON EXISTING TAC CONFIGURATION AND PROPOSED TAC CONFIGURATION

\begin{tabular}{|c|c|c|c|c|}
\hline \multirow{2}{*}{$\begin{array}{c}\text { Font } \\
\text { Size }\end{array}$} & \multicolumn{2}{|c|}{$\begin{array}{c}\text { Existing TAC } \\
\text { Configuration }\end{array}$} & \multicolumn{2}{c|}{$\begin{array}{c}\text { Proposed TAC } \\
\text { Configuration }\end{array}$} \\
\cline { 2 - 5 } & \# of Cells & PTL & \# of Cells & PTL \\
\hline 4300 & 119 & 857 & 46 & 68 \\
\hline 4301 & 77 & 830 & 120 & 1337 \\
\hline 4302 & 54 & 402 & 66 & 552 \\
\hline 4303 & 76 & 575 & 103 & 833 \\
\hline 4304 & 77 & 782 & 96 & 960 \\
\hline 4305 & 125 & 794 & 96 & 513 \\
\hline 4306 & 43 & 248 & 47 & 250 \\
\hline 4307 & 75 & 402 & 74 & 383 \\
\hline 4308 & 97 & 677 & 100 & 747 \\
\hline 4309 & 81 & 576 & 79 & 513 \\
\hline 430A & 39 & 97 & 41 & 112 \\
\hline 430B & 125 & 500 & 125 & 500 \\
\hline 430C & 198 & 410 & 198 & 410 \\
\hline 430D & 268 & 875 & 268 & 875 \\
\hline 430E & 156 & 346 & 151 & 318 \\
\hline Total & 1610 & 8371 & 1610 & 8371 \\
\hline PSP & & $\mathbf{7 0 . 2 6}$ & & $\mathbf{8 4 . 2 1}$ \\
\hline
\end{tabular}

For the existing TAC configuration in the table, the PSP calculated 70.26 . The proposed TAC configuration provides the PSP of $84.21 \%$, which is higher than the existing scheme by $13.95 \%$.

Because $\mathrm{S}_{\mathrm{TAC}}$ is defined as 120 cells, a cell in area does not move to the TAC, which consists of more than 120 cells. Table1 shows that all of cells in area dose not move to the TAC, which consists of more than 120 cells.

Figure 7 shows the improvement of PSP for Area Code 43. All the case, PSP improves more than existing TAC configuration. At the tabu size $=50$, PSP is improved to $120.48 \%$ and tabu size $=200$, PSP is improved to 119.85 That is, too large tabu size is inefficient to PSP improvement.

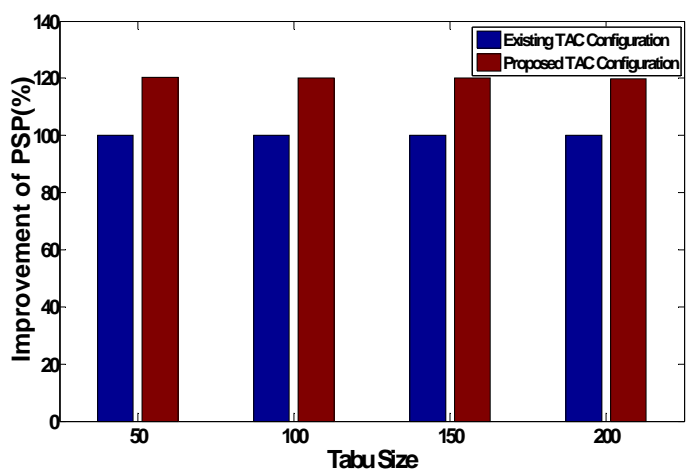

Figure 7. Improvement of PSP for Area 43
Figure 8 shows the geographical distribution of cells in the target area 27, in which the cells are depicted with the same shape for each of TACs. From the figure, we can see that the proposed scheme (Fig. 8(a)) gives more compact and balanced TAC configurations that the existing scheme (Fig. 8(b)). This is because the proposed scheme gives the TAC configuration by considering the feasibility conditions, while PSP is maximized
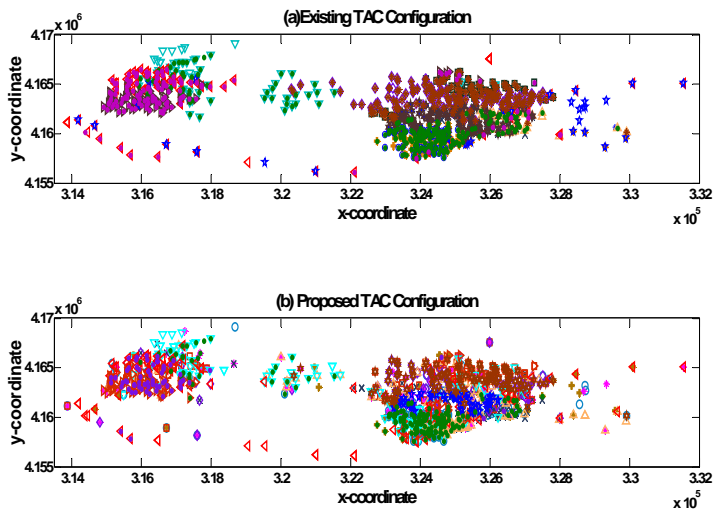

Figure 8. Geographical Distibution of TACs for Area 43

\section{CONCLUSIONS}

In this paper, we presented a new TAC configuration scheme to maximize the paging success rates in mobile communication systems, with tabu search.

By experimentations with real-world data of SK Telecom in Korea, the proposed scheme is compared with the existing scheme in terms of the paging success rate. From the results, we can see that the proposed scheme provides more optimized TAC configurations than the existing scheme by maximizing the paging success rate. It is also noted that the proposed scheme can give much more balanced TAC configurations, compared to the existing scheme.

\section{ACKNOWLEDGMENT}

This research was supported by the Basic Science Research Program of NRF(2010-0020926).

\section{REFERENCES}

[1] S Astély David, et al., "LTE: the evolution of mobile broadband," IEEE Communications Magazine, Vol. 47, No. 4 (2009): 44-51.

[2] 3GPP TS 36.300, Evolved Universal Terrestrial Radio Access (EUTRA) and Evolved Universal Terrestrial Radio Access Network (EUTRAN): Overall Description Stage 2, 2008.

[3] Lyberopoulos George L., et al., "Intelligent paging strategies for third generation mobile telecommunication systems," IEEE Transactions on Vehicular Technology, Vol. 44, No.3 (1995): 543-554.

[4] Bar-Noy Amotz, et al., "Paging mobile users in cellular networks: Optimality versus complexity and simplicity," Theoretical Computer Science, Vol. 470 (2013): 23-35.

[5] Richard Johnsonbaugh and Marcus Schaefer, Algorithms, Prentice Hall, 2004.

[6] Gendreau, Michel, and Jean-Yves Potvin. "Tabu search." Search methodologies. Springer US, 2005. 165-186. 
[7] Seok-Joo Koh, "A Tabu Search Approach for the Survivable Fiber Optic Communication Networks Design”, Department of Management Science, 1994.

[8] Seck-Joo Koh, "Design and Management of Survivable Communication Networks in B-ISDN Environments", Department of Industrial Engineering, 1998.

[9] F. Glover, "Tabu Search: A Tutorial”, Interfaces, Vol. 20, No.4 (1990), $74-94$.

[10] F. Glover, "Tabu Search - Part 1", ORSA Journal on Computing, Vol. 1 (1989), 190 - 206

[11] F. Glover, "Tabu Search - Part 2", ORSA Journal on Computing, Vol. 2 (1990), $4-32$.

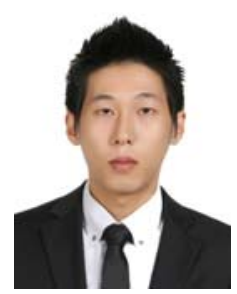

Hyung-Woo Kang received B.S degree in Computer Science from Kyungpook National University in 2011. He is now a Master course in School of Electrical Engineering and Computer Science from Kyungpook National University. His current research interests include mobile communication systems.

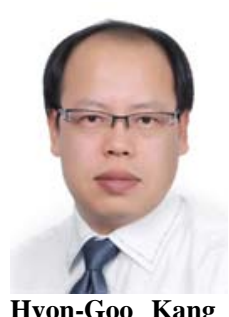

Hyon-Goo Kang received B.S. degree in Mathematics and M.S degrees in Industrial Engineering from KAIST in 1996 and 1998. He also received Ph.D. degree in Industrial Engineering in KAIST in 2003. From 2003 to 2011, he worked for WiMAX and LTE System Design and SW Development in Samsung Electronics. Since 2011, he has been with the Access Network Lab in SK Telecom, as an R\&D Manager, Ph.D. E-mail: hyongoo.kang@sk.com

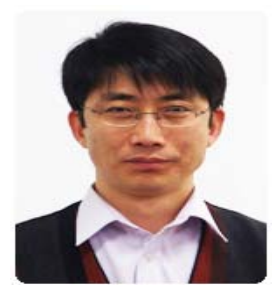

Seok-Joo Koh received B.S. and M.S. degrees in Management Science from KAIST in 1992 and 1994, respectively. He also received Ph.D degree in Industrial Engineering from KAIST in 1998. From August 1998 to February 2004, he worked for Protocol Engineering Center in ETRI. Since March 2004, he has been with the school of Electrical Engineering and Computer Science in the Kyungpook National University as an Associate Professor. His current research interests include mobility control in Future Internet, mobile SCTP, and mobile multicasting. He has also participated in the International Standardization as an editor in the ITU-T SG13 and ISO/IEC JTC1/SC6. 